\title{
Determination of Fatigue as Assessed by Mechanomyogram and Electromyogram Following Maximal Loaded Exercise in Exercise Trained Men
}

\author{
Gürkan Bilgin1, 5, İ. Ethem Hindistan², Y. Gül Özkaya², Etem Köklükaya³, Övünç Polat , Ömer H. \\ Çolak $^{4^{*}}$ \\ ${ }^{1}$ Vocational School of Technical Sciences, Mehmet Akif Ersoy University, Burdur, Turkey. \\ ${ }^{2}$ School of Physical Education and Sports, Akdeniz University, Antalya, Turkey. \\ ${ }^{3}$ Faculty of Engineering, Department of Electrical and Electronics Engineering, Gazi University, Ankara, \\ Turkey. \\ ${ }^{4}$ Faculty of Engineering, Department of Electrical and Electronics Engineering, Akdeniz University, Antalya, \\ Turkey. \\ ${ }^{5}$ Institute of Natural Science, Department of Electrical and Electronics Engineering, Sakarya University, \\ Turkey.
}

* Corresponding author. Tel.: 02423106361; email: omercol@akdeniz.edu.tr Manuscript submitted July 10, 2015; accepted October 15, 2015.

doi: 10.17706/ijcee.2015.7.5.305-315

\begin{abstract}
In this study, determination of fatigue at the onset, and following maximal loaded exercise was evaluated from mechanomyography (MMG) and electromyography (EMG) recordings in exercise trained men. Twenty four recreationally active men were participated into this study. Participants were performed a fatiguing Bruce Protocol which is widely-used maximal loading treadmill test. EMG and MMG recordings were obtained from rectus femoris muscle during maximal isometric contractions both at the onset, and following Bruce Protocol. Following pre-training measurements, volunteers were subjected to sprint running exercise involved 3 sessions per week for 8 weeks. MMG and EMG recordings were repeated following 8 weeks of sprint running training. Recordings were decomposed in 8 levels by using the wavelet packet transform and so windows with 15 nodes and 5 sliding nodes were created. Then the mean energy values at each window were obtained from the 24 persons group and were applied to the input of Multilayer Perceptron Neural Network (MLPNN). In the output, the classification process was performed both before and after fatiguing exercise, and the success rate of the test was evaluated for fatigue. In the post-training measurements, MMG has been failed to determine fatigue with decreased success rate, whereas EMG results were shown that success rate is increased by 20 percent compared to pre-training measurements.
\end{abstract}

Key words: Electromyogram (EMG), fatigue, mechanomyogram (MMG), wavelet packet transform.

\section{Introduction}

Physical fatigue or muscle fatigue is the decrease in ability of a muscle to generate force. The determination of muscle fatigue adds important information to the fields of sport performance and injuries. Owing to the changeability of the muscle characteristics from person to person, there is no simple function of muscle load and timing which defines a precise muscle fatigue threshold [1]. Usually, researchers have used techniques that affected by the performance and activity of whole body. These include changes in the 
lactate production, respiratory oxygen consumption and carbon dioxide emission amount, ECG (electrocardiogram) measurements, et cetera. At the present time, the studies of detecting the acute fatigue in the muscles gradually increase. Myoelectric indications of muscle fatigue can be determined by measuring electric signal in the muscle and refer to changes in amplitude and signal frequency and in the muscle conduction velocity (CV), while the mechanical factors relate to a loss in the applied force [2]. EMG (Electromyogram) is one of these methods which is often used in identifying muscle fatigue. Previous studies demonstrated that muscle fatigue can be determined by using several parameters such as variations in action potential conduction velocity, variations of EMG amplitudes and inclining to low frequencies at power spectrum analysis [3]-[9]. Muscle fatigue was also estimated by calculating of mean power frequency of EMG recordings and then pursuant to the results of a previous study, a sliding from the high frequency spectrum to low frequency spectrum was observed when the muscle is fatigued [10]. In a further study, researchers were tried to estimate the amount of fatigue during the static muscle contraction by using analysis of variance of surface electromyogram (sEMG) [11].

The other method for determination of muscle fatigue is mechanomyogram (MMG). The recordings are taken on the skin of the involved muscles when the muscle is contracted. Generally the accelerometer uses for signal records of MMG. MMG can be described which the sum of the mechanical activity of the individual motor units as EMG [12]. Shinohara et al. evaluated the MMG behavior of the quadriceps muscles during increasing power output and rising up to the maximal bicycle ergometer and the results were compared with when the SEMG and analysis demonstrated that a linear correlation between the MMG of the quadriceps muscle and work load during the maximal incremental cycle ergometer [13]. A study was done scientific study to detect the correlation between MMG, EMG and Torque records of quadriceps muscles response. The study results indicated that the MMG amplitude may be useful for estimating force production during fatiguing dynamic contractions [14]. Beck et al. have tried to solve time-frequency events of MMG signals obtained from vastus lateralis, rectus femoris and vastus medialis muscles by using nonlinear scaling of the wavelet during maximal movements. They determined different spectral bands for maximal movements on MMG [15].

The purpose of the present study is to determine muscle fatigue following a maximal exercise loading in exercise trained persons. We used wavelet packet transform and MLPNN to analyze the MMG and EMG recordings obtained by surface electrodes of thigh muscle.

\section{Methods}

\subsection{Participants}

Twenty four healthy, recreationally active men aged 18-23 years were voluntarily participated into this study. The exercise training program and all measurements were performed in Akdeniz University, Sport Science Research and Application Center. The inclusion criteria were non-user of alcohol, without medical illness, non-smoker, the body mass index (BMI) ranged 20 to 25, not receiving medication or vitamins, and willing to participate. In the selection of volunteers, it was ensured that the participants do not to exercise at least one week before the experimental period. The subjects provided informed consent, and a standardized individual information session was organized by an exercise specialist to instruct the participants on how to apply the exercise session. Descriptive information for the participants are presented at Table 1.

\subsection{Exercise Training}

All participants were performed repeated maximal sprints, after completion of a 20-minute warm-up. An outdoor track was used that was covered with tartan surface. The participants performed 4 sets of 4 
maximal repetitions of $25-\mathrm{m}$ sprints 3 times a week, where the active recovery time was 2.5 minutes between repetitions, and 5 minutes between sets which was sufficient for the participants to recover fully [16]. This training program continued until the fourth week. For each of the remaining 2 weeks, 2 repetitions were added to first and third sets, and finally, additional 2 repetitions were added to second and four sets at the 7 th and 8 th weeks. As a result, exercise training was completed as 4 sets of 5 repetitions at the end of the 8 weeks of training program.

Table 1. The Arrangement of Channels

\begin{tabular}{|l|l|}
\hline Age (years) & $19,93 \pm 1,64$ \\
\hline Height $(\mathrm{cm})$ & $174,78 \pm 7,2$ \\
\hline Body mass $(\mathrm{kg})$ & $66,35 \pm 6.81$ \\
\hline Body mass index $\left(\mathrm{kg} \cdot \mathrm{m}^{-2}\right)$ & $21,72 \pm 1,74$ \\
\hline Fat $\%$ & $10,93 \pm 2,81$ \\
\hline Fat mass $(\mathrm{kg})$ & $7,35 \pm 2,31$ \\
\hline Fat free mass $(\mathrm{kg})$ & $59,03 \pm 5,48$ \\
\hline Total Body Water $(\mathrm{kg})$ & $43,23 \pm 4,1$ \\
\hline
\end{tabular}

\subsection{Assessment}

Height was measured using an ultrasonic height measure (Soehnle-Waagen GmbH \& Co. KG). Body weight, $\%$ fat, fat mass, fat-free mass and total body water were measured by using a Tanita Body Composition Analyzer (Model TBF-300 TANITA, Tokyo, Japan). Body mass index (BMI) was calculated from weight and height.

Margaria Kalamen staircase test was performed to measure anaerobic capacity of participants [17]. All subjects were weighed without shoes and in minimal clothing. The Margaria Kalamen test is characterized by a subject sprinting up a staircase of specified height from a specific distance, stepping only on the third, sixth and ninth steps. Bosco New Test 2000 model (FIN) photocells were placed on steps 3 and 9. The time started at initial contact with the third step and stopped at contact with the ninth step. Subjects were instructed to complete the sprint up the stairs with maximum velocity. The total power produced during the test was calculated by the following formula,

$$
\operatorname{Power}(\mathrm{w})=\frac{\text { weight }(\mathrm{kg}) \times 9,8 \mathrm{~ms}^{-1} x \text { distance }(\mathrm{m})}{\text { time }(\mathrm{s})}
$$

Aerobic capacity of all participants were measured by using gas analyser during Bruce treadmill protocol. Expired gases were analysed during all tests using an automated on-line metabolic analysis system (Sensor Medics, CA), in the breath by breath mode. The Sensor Medics Paramagnetic $\mathrm{O}_{2}$ analyzer and $\mathrm{CO}_{2}$ analyzer were calibrated before and after each test by using two precision reference gases of known concentrations. $\mathrm{The} \mathrm{VO}_{2}$ max values as an index of aerobic capacity are expressed in $\mathrm{ml} \cdot \mathrm{kg}^{-1} \cdot \mathrm{min}^{-1}$.

\subsection{Experimental Scheme}

Following resting EMG and MMG recordings, all participants were performed the Bruce protocol which is a widely-used gradually maximal-loaded treadmill method to occur fatigue. In Bruce protocol, the volunteers were expected to give the maximum performance during increasing speed and ramp level of the treadmill at certain time intervals. The time-dependent changes in the speed and ramp levels during Bruce Protocol were given below Table 2 . 
Table 2. Bruce Protocol

\begin{tabular}{|c|c|c|c|}
\hline \multicolumn{4}{|c|}{ Time-dependent changes in the speed and ramp levels according to } \\
Bruce Protocol \\
\hline Steps & Time (Minutes) & Km/h & Ramp (Degree) \\
\hline 1 & 0 & 2,74 & 10 \\
\hline 2 & 3 & 4,02 & 12 \\
\hline 3 & 6 & 5,47 & 14 \\
\hline 4 & 9 & 6,76 & 16 \\
\hline 5 & 12 & 8,05 & 18 \\
\hline 6 & 15 & 8,85 & 20 \\
\hline 7 & 18 & 9,65 & 22 \\
\hline 8 & 21 & 10,46 & 24 \\
\hline 9 & 24 & 11,26 & 26 \\
\hline 10 & 27 & 12,07 & 28 \\
\hline
\end{tabular}

In this study, EMG and MMG recordings were obtained from each subject for analysis at the onset (volunteers who are not fatigued), and following Bruce protocol (fatigued volunteers). Afterwards, all volunteers have participated in the repeated sprint training 3 days per week for 8 weeks. At the end of the sprint training program, EMG and MMG recordings were repeated from each subject for analysis at the onset, and following Bruce protocol.

For EMG recordings, a surface electrode was fixed as on the belly of the rectus femoris (RF) muscle of dominant thigh of the volunteers. Additionally, simultaneous recordings of MMG and EMG were taken by placing accelerometer above the center of the RF muscle.

Following a 10 min warm-up period, volunteers were asked to sit in a chair with hips and knees flexed at $90^{\circ}$ with the arms were crossed with hands placed on the opposite shoulder. Each subject performed maximal voluntary isometric fatiguing contractions with dominant knee extensor muscles. Periodic metronome was used to stability of contractions at $1 \mathrm{~Hz}$.

Surface EMG recordings were obtained from ProComp Infiniti TM (Model SA7500, Thought Technology Ltd. 2180 Belgrave Avenue, Montreal, QC H4A 2L8 Canada) EMG recorder by using self-adhesive, $10 \mathrm{~mm}$ in diameter, silver-silver chloride triode electrodes (T3404, Thought Technology Ltd. Montreal, Canada) in the laboratory environment. Before the recordings, the skin surface where the electrodes are placed were cleaned and shaved in order not to cause any unwanted noises. In order to obtain a better signal, conductive gel was used between the electrode and the skin surface.

Accelerometer and all equipment used in the experiments for MMG recordings were purchased from Company Benair [(Beandevice AX-3D wireless accelerometer, Bean Gateway wireless sensor Networks (WSN) coordinator (wireless sensor network coordinator), Beanscape Real time wireless sensor networks supervision (real-time wireless sensor network control software)].

\subsection{Wavelet Packet Transform}

As a modified version of the standard wavelet, the Wavelet Packet Transform (WPT) defines a generalization of the multi-resolution analysis and use the entire family of sub-band decomposition. This multi-resolution analysis technique has been used with good results in many studies, such as biomedical signal classification [18], detection [19] etc.

Differently from classical wavelet transform which is realized only by a low-pass filter, wavelet packet transform is implemented by a basic two-channel filter (low-pass and high-pass filters). Using the WPT, the information in high frequencies can be analyzed as well as that in low frequencies and as a result, finer frequency bands can be gained by WPT than by wavelet transform.

In the analysis made by using the discrete wavelet transform (DWT), the signal must be decomposed into coefficients at different levels. In the DWT analysis at all levels decomposition process is applied only approximation coefficients. Wavelet packet transform (WPT) is a special form of DWT, which offers more 
potential decomposition. Differently from DWT, decomposition processes are applied to both approximation and detail coefficients. The decomposition process of coefficients is performed by using low and high pass filters. Approximation coefficients in the low-pass filter output and detail coefficients in the high-pass filter output are obtained. WPT mathematically can be described as,

$$
\begin{gathered}
W_{2 j}(t)=\sqrt{2} \sum_{k=0}^{2 j-1} h(k) W_{j}(2 t-k) \\
W_{2 j+1}(t)=\sqrt{2} \sum_{k=0}^{2 j-1} g(k) W_{j}(2 t-k)
\end{gathered}
$$

As in (1) and (2), $h(k)$ and $g(k)$ are implemented as a high pass filter and low-pass filter coefficients. $W_{2 j}(t)$ is defined as a high-pass filter as in (1), and $W_{2 j+1}(t)$ is defined as a low-pass filter as in (2). In here, $W_{0}(t)=\phi(t)$ refers to the scaling function. $W_{1}(t)=\psi(t)$ symbolizes the wavelet function [20].

If $W_{j}(t)$ is considered as a three indexed function,

$$
W_{m, j, n}(t)=2^{-m / 2} W_{j}\left(2^{-m} t-n\right)
$$

where $j \in N,(m, n) \in Z^{2}$ and " $j$ " is the node index in each level, " $n$ " is the shifting parameter in time axis and " $m$ " is the scaling parameter.

The mean energy values of nodes are calculated as

$$
W_{\text {mean_energy }, m, j}=\frac{1}{N} \sum\left|W_{m, j}(r)\right|^{2}
$$

" $r$ " is the interested number of data and " $N$ " total number of data in $m$ th level.

The frequency values of WP can be found follows,

$$
f_{m}=\frac{(j+1) f_{s}}{2^{m+1}}, m=1, \ldots, M-1
$$

In here, " $f_{s}$ " is sampling frequency, " $f_{m}$ " is frequency in $m$ th level. Interval of " $j$ " is indicated as $j=0,1, \ldots, 2^{m}-1$ for WP [21], [22].

WP decomposition tree can be created as shown in Fig. 1 with $W_{m, j}$ indexes.

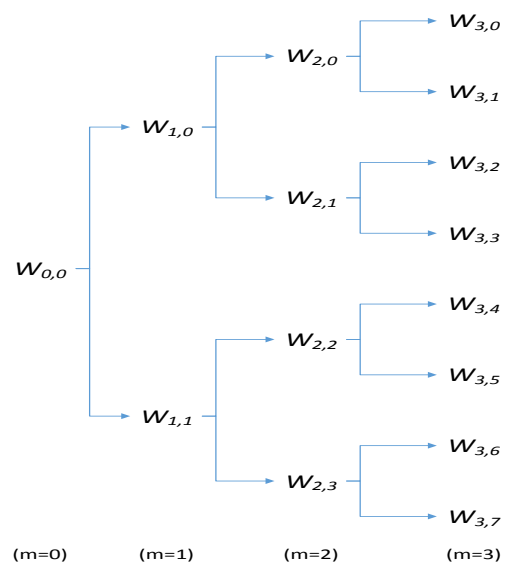

Fig. 1. The Decomposition of the wavelet packets depending on level. 


\subsection{Multilayer Perceptron Neural Network (MLPNN)}

The purpose of this study is the determination of fatigue following Bruce protocol. A specific network structure is chosen for this study. This specific feed forward neural network consists of four layers that contain an input layer, two hidden layers with 10 and 20 neurons, and output layer with one neuron. The Levenberg-Marquardt (LM) algorithm is used as the training algorithm.

The structure of Multilayer Perceptron Neural Network for this study is shown in Fig. 2.

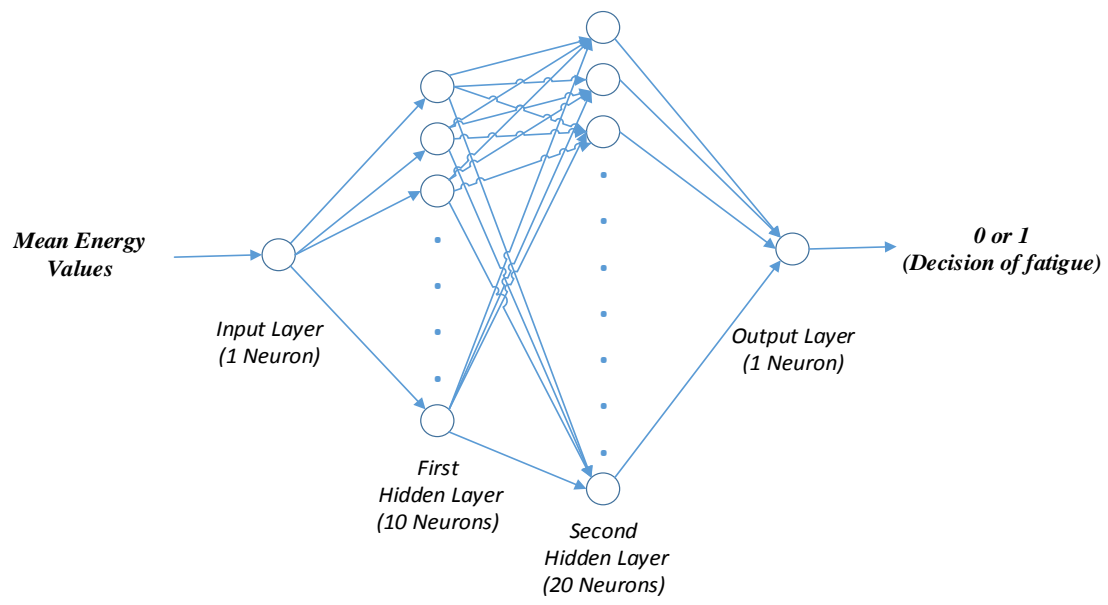

Fig. 2. The feed forward neural network structure for the estimation of acute muscle fatigue.

\subsection{Data Processing}

\subsubsection{Pre-processing}

In the first instance obtained EMG and MMG records were passed through a pre-processing stage in order to make ready for analysis. In this pre-processing stage, EMG values taken at the $2 \mathrm{kHz}$ sampling frequency were filtered through $50 \mathrm{~Hz}$ notch filter and this process was applied to all harmonics up to the 14th. Afterwards EMG recordings have been made ready for analysis by passing the second degree FIR filter in the 3-450 $\mathrm{Hz}$ band. In the pre-processing part of MMG recordings, they were re-sampled to $2 \mathrm{kHz}$ by using cubic spline interpolation.

\subsubsection{Analysis}

In this study, WPT was used for feature extraction of EMG and MMG. EMG and MMG recordings were decomposed in 8 levels. At the end of the 8th level, 256 nodes occurred and each energy value of the node was calculated for 24 volunteers. The energy of each node was normalized by dividing the energy values to the maximum energy value for each volunteer. Normalized energy matrix of MMG and EMG was created at size $24 \times 256$. A window length which is long enough to cover 15 nodes was selected. This window was slid 5 nodes along the 10th-115th node range (39-450 Hz frequency range) and the mean energy value of the nodes were calculated in the remaining windows for each volunteers as in Fig. 3. Then, a multilayer neural network was designed which the input values was chosen as this calculated average energy values.

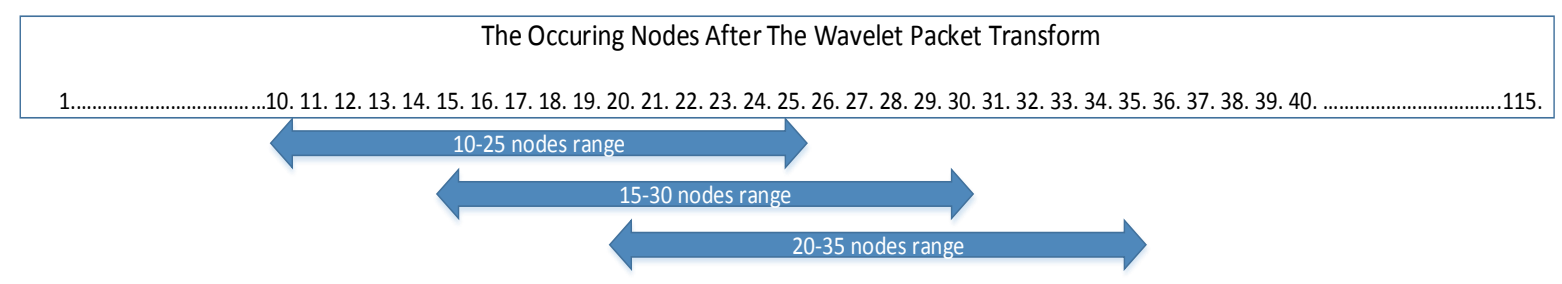

Fig. 3. Sliding windows. 
The output was defined as "0" before Bruce Protocol and as "1" after the Bruce Protocol. In the analysis, 14 records were used for training data, and the remaining 10 records were used as test data.

At first, the average window energy values of obtained from the EMG group are given to the input of the network. Training and test success rates were evaluated in the end of the process. The same procedure was also repeated for MMG data. The flow chart of this study was shown in Fig. 4.

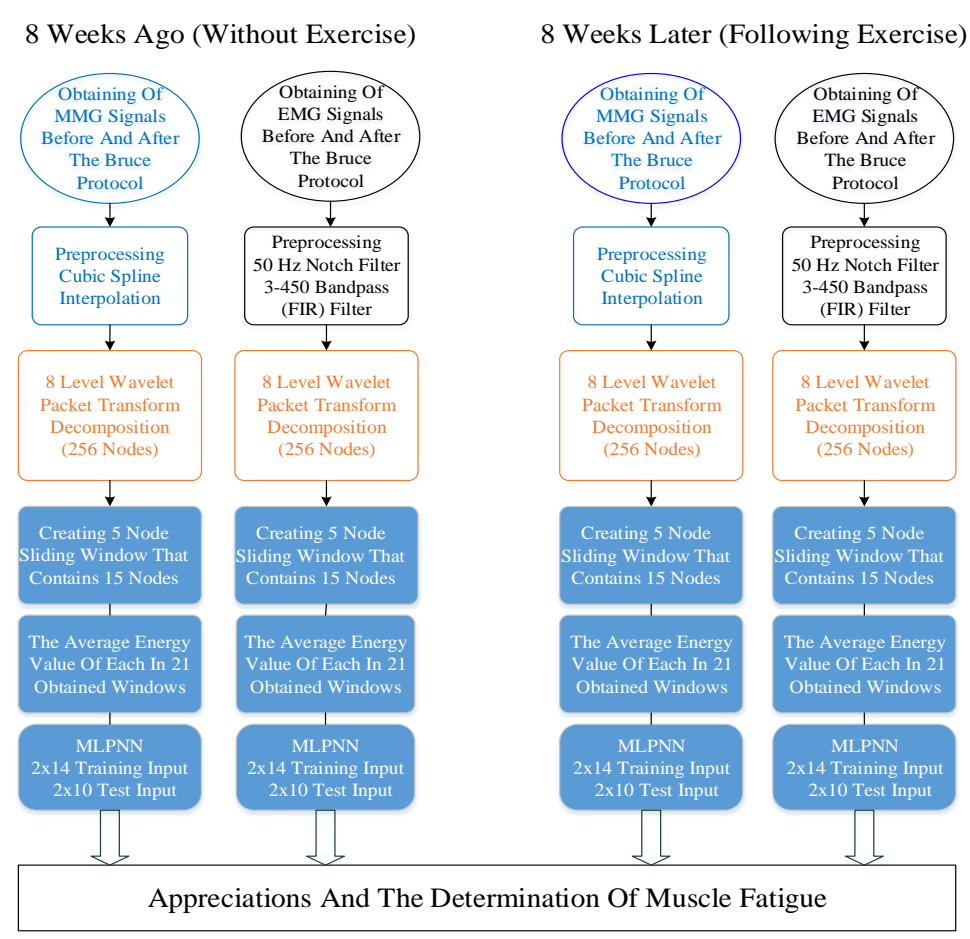

Fig. 4. Flow chart of this study.

\subsection{Statistical Analysis}

Data are expressed as means +S.D., and the statistical significance of the aerobic and anaerobic capacity of the pre- and post-training measurements were assessed by t-test. A level of $p<0.05$ was accepted as statistically significant.

\section{Results}

The aerobic and anaerobic capacities of participants were presented at Table 3. Repeated sprint training for 8 weeks resulted significantly increased aerobic $(p<0.001)$ and anaerobic $(p<0.05)$ capacity of all volunteers.

Table 3. Pre- and Post-training Aerobic and Anaerobic Capacity Results of Participants

\begin{tabular}{|c|c|c|}
\hline & $\begin{array}{c}\text { Aerobic Capacity } \\
\text { (ml.kg-1.dk } \text {. }^{-1}\end{array}$ & $\begin{array}{c}\text { Anaerobic Capacity } \\
\text { (Watts) }\end{array}$ \\
\hline Pre-training & $50,20 \pm 6,031$ & $1389 \pm 191$ \\
\hline Post-training & $61,34 \pm 10,49^{* * *}$ & $1433 \pm 187^{*}$ \\
\hline
\end{tabular}

Values are expressed as mean $+\mathrm{SD},{ }^{*} p<0.05,{ }^{* * *} p<0.001$, significant difference from pre-training measurement.

In pre-training measurements obtained from the MMG recordings, the achieved highest test success rate was $77 \%$, and overall test successes rates were found between $65 \%$ and $77 \%$. In the fatigue analysis, test success rates were determined between $63 \%$ and $77 \%$ by using the EMG recordings alone taken from the same people. The test success rates over 75\% were seen in $136.72 \mathrm{~Hz}-195.31 \mathrm{~Hz}, 234.37 \mathrm{~Hz}-292.97 \mathrm{~Hz}$, $273.44 \mathrm{~Hz}-332.03 \mathrm{~Hz}, 292.97 \mathrm{~Hz}-351.56 \mathrm{~Hz}, 312.50 \mathrm{~Hz}-371.09$ frequency ranges. After 8 weeks of sprint 
training, a significant decrease was observed in the muscle fatigue analysis using just MMG recordings, and test success rates were found between $55 \%$ and $65 \%$. The bands that having high success rate $(65 \%)$ exhibited differences from the bands of pre-training measurements. The test success rate reached $90 \%$ in the muscle fatigue analysis with EMG records obtained from trained volunteers. The successes in 273.44 $\mathrm{Hz}-332.03 \mathrm{~Hz}$ and $332.03 \mathrm{~Hz}-390.62 \mathrm{~Hz}$ frequency ranges were $85 \%$ and $90 \%$. The success rate in the present study showed an alteration between $70 \%$ and $90 \%$. Thus, in exercise trained person, MMG data will not be alone decisive for determining fatigue and EMG data has been demonstrated to operate better performance in determining fatigue.

\section{Discussion}
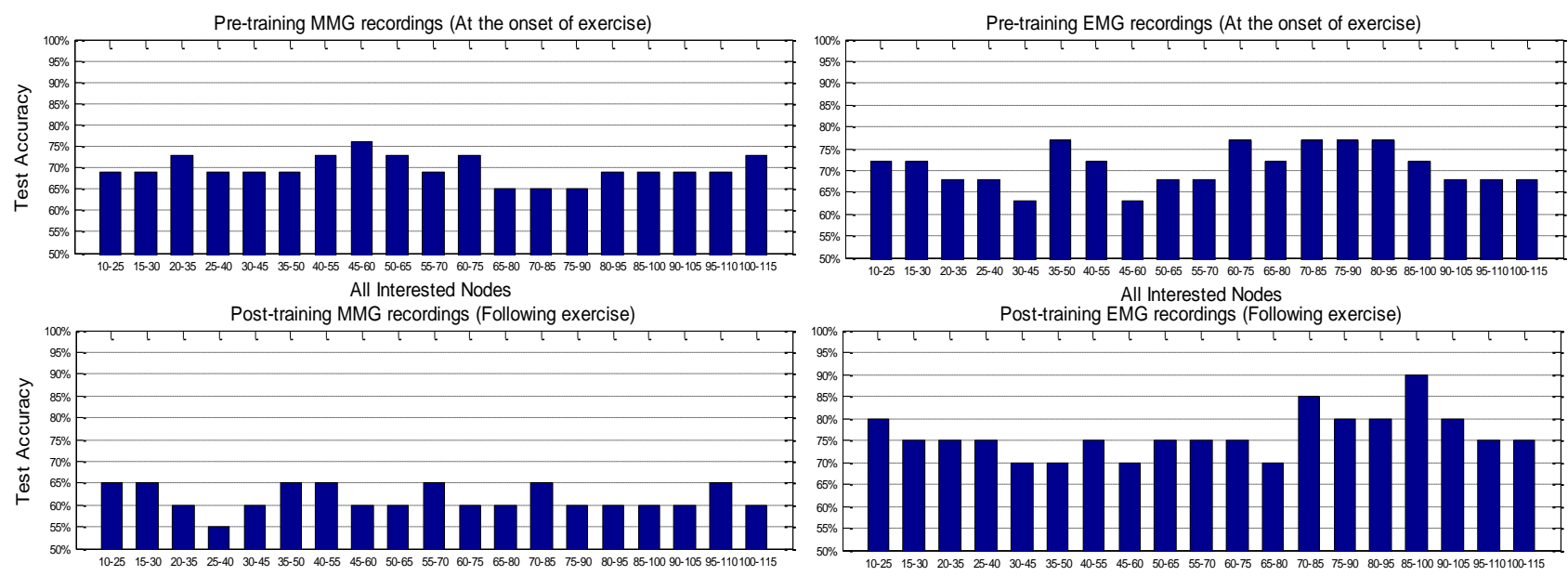

All Interested Nodes

All Interested Nodes

Fig. 5. a). The test accuracy rates by using MMG records analysis for determination of fatigue in pre (at the onset of exercise) and post (following exercise) exercise training. b). The test accuracy rates by using EMG records analysis for determination of fatigue in pre (at the onset of exercise) and post (following exercise)

exercise training.

This study evaluated the usage of MMG and EMG as a tool on detection of fatigue following maximal exercise loading in exercise trained men. The results of the present study clearly demonstrated that MMG and EMG recordings are useful, and detectable tools in pre-training period. However, in exercise trained state, MMG fails to detect fatigue, although EMG becomes a more powerful method following exercise training.

In the present study, MMG recordings were obtained during maximal isometric fatiguing contractions by using an accelerometer which detects muscle displacements in three dimensions. In contrast to EMG, MMG reflects both motor unit activation strategy and contractile properties of motor units [23].

In the Fig. 5 seen that MMG records cannot be alone determinative for detecting of fatigue but EMG records have been demonstrated to operate better performance in detecting of fatigue. After 8 weeks of sprint training, the test success rates were found between $55 \%$ and $65 \%$ by using MMG recordings analysis, but the test success rates in the same study showed an alteration between $70 \%$ and $90 \%$ by using EMG recordings.

Motor unit recruitment and discharge rate are two main factors in an increased muscle force in voluntary contractions [12], [23]. In the fatiguing isometric contractions, MMG alterations has been shown to persist in low to high intensity voluntary contractions [24]. In the previous studies, increased motor unit recruitment and decreased discharge rate of motor units has been shown to increase MMG activity [23]. In contrast, decreased motor unit discharge rate has been shown to decrease EMG activity. Our results suggest that motor unit activation strategy is one of the prominent factor for the determination of fatigue during 
maximal fatiguing contractions.

In the present study, although we did not intend, or measure muscle hypertrophy or muscle force, increased aerobic and anaerobic capacity values of participants has been showed that the training-induced adaptations has been effectively achieved. Limonta et al. investigated motor unit activation pattern in climbers, and showed increased EMG and MMG activities following fatiguing contractions. The researcher concluded that higher force generation capacity of climbers is attributed to increased number of large and less fatigable motor units [25]. The conflicting results could be due to the methodological differences, since in our study, participants were applied an exercise training program lasted in 8 weeks, in contrast to the participants climbing for several years in the study conducted by Limonta et al. [25].

The original finding of the present study is that the MMG has been failed to detect fatigue following repeated sprint training could be resulted from several physiological adaptations. One of these adaptations could be altered motor unit activation strategy following exercise training. As mentioned previously, there is an inverse correlation between MMG activity and discharge rate of motor units [23]. On the other hand, Yosithake et al. demonstrated that, when the discharge rate of motor units increase, force fluctuations result a decrement on MMG activity [26].

Another potential mechanism for explaining the finding that MMG has been failed to detect following exercise is altered intrinsic mechanical properties of muscles following exercise training. Blazevich et al. have showed the altered architecture of quadriceps muscle with an increase in fascicle length following 10 weeks of resistance training [27].

Several limitations should be mentioned into this study. We did not measure muscle strength which could give additional information to distinguish motor unit activation patterns and contractile properties of muscles following fatiguing contractions. We also did not measure antagonist muscle activities which could be given additional information on motor unit activation pattern following fatiguing exercise. Future research on different exercise training methods such as aerobic, resistance, or complex exercise could be conduct to elucidate the motor unit behavior following fatiguing exercise.

\section{Conclusion}

In conclusion, the present study demonstrated that whereas MMG failed, EMG is a useful tool to detect fatigue following maximal loaded exercise in exercise trained state.

\section{Acknowledgment}

The research has been supported with project number: 2014.01.0102.001 by the Research Project Department of Akdeniz University, Antalya, Turkey. This study was approved as ethically by Akdeniz University, Faculty of Medicine, Scientific Research Assessing Authority with date/number:21.12.2010/220.

\section{References}

[1] Kumar, S., \& Mital, A. (1996). Electromyography in Ergonomics. London, UK: Taylor and Francis Ltd, Publisher.

[2] Asghari, O. M., Hu H., \& Gan J. Q. (2008). Manifestation of fatigue in myoelectric signals of dynamic contractions produced during playing PC games. Proceedings of the 30th Annual International IEEE EMBS Conference (pp. 315-318). Vancouver, BS, Canada.

[3] Comi, G., Leocani, L., Rossi, P., \& Colombo, B. (2001). Physiopathology and treatment of fatigue in multiple sclerosis. J. Neurol, 248, 174-179.

[4] Mathur, S., Eng, J. J., \& MacIntyre, D. L. (2005). Reliability of surface EMG during sustained contractions of the quadriceps. Journal of Electromyography and Kinesiology, 15, 102-110.

[5] Strimpakos, N., et al. (2005). Issues in relation to the repeatability of and correlation between EMG and 
Borg scale assessments of neck muscle fatigue. J. of Electromyography and Kinesiology, 15 (5), 452-465.

[6] Ravier, P., Buttelli, O., Jennane, R., \& Couratier, P. (2005). An EMG fractal indicator having different sensitivities to changes in force and muscle fatique during voluntary static muscle contractions. Journal of Electromyography and Kinesiology, 15, 210-221.

[7] De Luca, C. J. (1997). The use of surface electromyography in Biomech. J. Appl. Biomech, 13, 135-163.

[8] Lindstrom, L., Kadefors, R., \& Petersen, I. (1977). An electromyographic index for localized muscle fatigue. J. Appl. Physiol, 43, 750-754.

[9] Marras, W. S. (1990). Industrial electromyography (EMG). Int. J. Ind. Ergon, 6, 89-74.

[10] Sakurai, T., et al. (2010). Detection of muscle fatigue by the surface electromyogram and its application. Proceedings of 9th IEEE/ACIS Int. Conference on Computer and Information Science (pp. 43-47).

[11] Soo, Y., et al. (2009). Quantative estimation of muscle fatigue using surface electromyography during static muscle contraction. Proceedings of Annual Int. Conference of the IEEE EMBS (pp. 2975-2978).

[12] Orizio, C., et al. (2003). The surface mechanomyogram as a tool to describe the influence of fatigue on biceps brachii motor unit activation strategy. Eur J. Appl Physiol, 90(3-4), 326-336.

[13] Shinohara, M., Kouzaki, M., et al. (1997). Mechanomyography of the human quadriceps muscle during incremental cycle ergometry. Eur. J. Appl. Physiol. Occup. Physiol, 76, 314-319.

[14] Perry-Rana, S. R., Housh, et al. (2002). MMG and EMG responses during fatiguing isokinetic muscle contractions at different velocities. Muscle Nerve, 26, 367-373.

[15] Beck, T. W., et al. (2008). Time/frequency events of surface mechanomyographic signals resolved by nonlinearly scaled wavelets. Biomedical Signal Processing and Control, 3(3), 255-266.

[16] McArdle, W. D., Katch, F. I., \& Katch, V. L. (2010). Exercise Physiology: Nutrition, Energy, and Human Performance. Lippincott Williams \& Wilkins.

[17] Huskey, T., Mayhew, J. L., Ball, T. E., \& Arnold, M. D. (1989). Factors affecting anaerobic power output in the Margaria-Kalamen test. Ergonomics, 32(8), 959-965.

[18] Behroozmand, R., \& Almasganj, F. (2007). Optimal selection of wavelet-packet-based features using genetic algorithm in pathological assessment of patients' speech signal with unilateral vocal fold paralysis. Computers in Biology and Medicine, 37(4), 474-485.

[19] Chendeb, M., Khalil, M., \& Duchêne, J. (2006). Methodology of wavelet packet selection for event detection. Signal Processing, 86(12), 3826-3841.

[20] Misiti, M., Misiti, Y., Oppenheim, G., \& Poggi, J. (1997-2002). Wavelet toolbox for use with MATLAB. The Mathworks Inc. User's Guide.

[21] Bilgin, S., Çolak, O. H., Koklukaya, E., \& Arı, N. (2008). Efficient solution for frequency band decomposition problem using wavelet packet in HRV. Digital Signal Processing, 18(6), 892-899.

[22] Bilgin, S., Çolak, O. H., Polat, O., \& Koklukaya, E. (2010). Determination of a new VLF band in HRV for ventricular tachyarrhythmia patients. Journal of Medical Systems, 34(2), 155-160.

[23] Shinohara, M., \& Søgaard, K. (2006). Mechanomyography for studying force fluctuations and muscle fatigue. Exercise and Sport Sciences Reviews, 34(2), 59-64.

[24] Blangsted, A. K., Sjøgaard, G., Madeleine, P., Olsen, H. B., \& Søgaard, K. (2005). Voluntary low-force contraction elicits prolonged low-frequency fatigue and changes in surface electromyography and mechanomyography. Journal of electromyography and kinesiology, 15(2), 138-148.

[25] Limonta, E., et al. (2015). Motor unit activation strategy during a sustained isometric contraction of finger flexor muscles in elite climbers. Journal of Sports Sciences, 1-10.

[26] Yoshitake, Y., et al. (2002). Characteristics of surface mechanomyogram are dependent on development of fusion of motor units in humans. Journal of Applied Physiology, 93(5), 1744-1752.

[27] Blazevich, A. J., et al. (2007). Influence of concentric and eccentric resistance training on architectural 
adaptation in human quadriceps muscles. Journal of Applied Physiology, 103(5), 1565-1575.

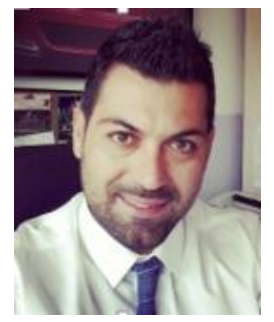

Gurkan Bilgin received the B.S. degree in 2007 and the M.S. degree in 2009 from the Department of Electrical and Electronic Engineering, Sakarya University, Turkey. He is a Ph.D. student in same university and he is working about signal processing.

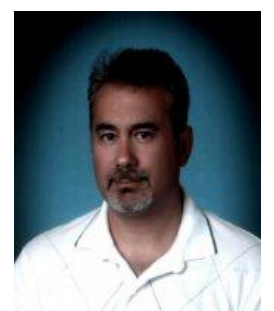

İ. Etem Hindistan received the B.S. degree in 1992 and the M.Sc. degree in 1995 from the Dept. of Physical Education Teaching, Marmara University,. He received his Ph.D. degree in 2015 from the School of Physical Education and Sports, Akdeniz University. He is a member of Akdeniz University, School of Physical Education and Sports. His research area is sport sciences.

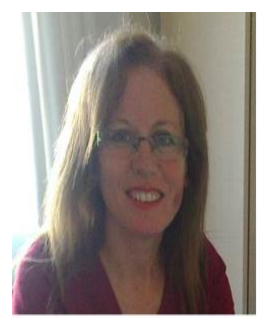

Y. Gul Ozkaya got the M.D. degree in 1994 from Akdeniz University, Faculty of Medicine. She is a member of Akdeniz University, School of Physical Education and Sports as an associate professor. Her research areas are exercise physiology and physiology.

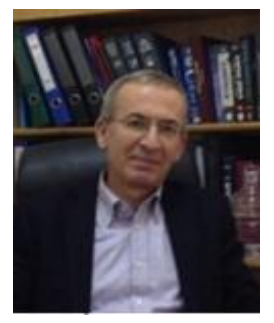

Etem Koklukaya received the B.S. degree in 1978 from I.T.U. Istanbul, Turkey in electronics and communication engineering, the M.S. degree in 1982 from Y.T.U. Istanbul, Turkey, in electronics and communication engineering, and the Ph.D. degree from I.T.U. in 1990 Istanbul, Turkey, in control and computer engineering. He has been a member of Gazi University, Turkey, as a professor at the Electrical and Electronics Engineering Department. His research areas are biomedical signal processing, control systems and artificial intelligence.

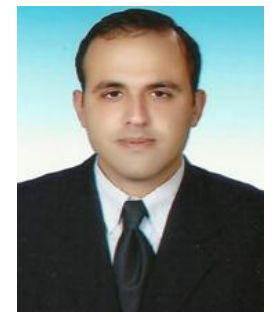

Ovunc Polat received the B.S. degree in 1999 and M.S. degree in 2003 from S.D.U. Isparta, Turkey, in electronics and communication engineering and the Ph.D. degree in 2007 from Yildiz Technical University, Turkey, in electrical and electronics engineering. He has been a member of Akdeniz University, Turkey, as an assistant professor at the Electrical and Electronics Engineering Department. His research areas are pattern recognition, signal detection and classification and image processing.

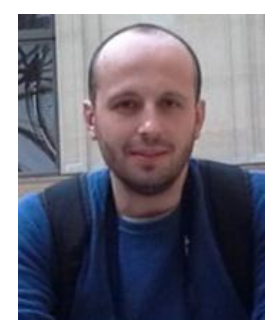

Omer H. Çolak received the B.S. degree in 1999 and the M.S. degree in 2003 from S.D.U. Isparta, Turkey, in electronics and communication engineering and the Ph.D. degree in 2006 from Sakarya University, Adapazari, Turkey, in electrical and electronics engineering. He has worked as an postdoctoral researcher in the Department of R\&D, University of Technology Zurich in 2008 and Universite Rene Descartes, Medicine Faculty, Paris in 2011. He has been a member of Akdeniz University, Turkey, as an associate professor at the Electrical and Electronics Engineering Department. His research areas are time frequency signal analysis, wavelet transform and applications, bioelectrical signal processing, neuroscience signal detection and classification. 